\title{
National indicators of health literacy: ability to understand health information and to engage actively with healthcare providers - a population- based survey among Danish adults
}

Anne Bo ${ }^{1}$, Karina Friis ${ }^{2}$, Richard $\mathrm{H}$ Osborne $^{3}$ and Helle Terkildsen Maindal ${ }^{1 *}$

\begin{abstract}
Background: Health literacy is a multidimensional concept covering a range of cognitive and social skills necessary for participation in health care. Knowledge of health literacy levels in general populations and how health literacy levels impacts on social health inequity is lacking. The primary aim of this study was to perform a population-based assessment of dimensions of health literacy related to understanding health information and to engaging with healthcare providers. Secondly, the aim was to examine associations between socio-economic characteristics with these dimensions of health literacy.
\end{abstract}

Methods: A population-based survey was conducted between January and April 2013 in the Central Denmark Region. Postal invitations were sent to a random sample of 46,354 individuals $>25$ years of age. Two health literacy dimensions were selected from the Health Literacy Questionnaire $\left(H \mathrm{LQ}{ }^{T M}\right)$ : i) Understanding health information well enough to know what to do (5 items), and ii) Ability to actively engage with health care providers (5 items). Response options ranged from 1 (very difficult) to 4 (very easy). We investigated the level of perceived difficulty of each task, and the associations between the two dimensions and socio-economic characteristics.

Results: A total of 29,473 (63.6\%) responded to the survey. Between 8.8\%, 95\% Cl: 8.4-9.2 and 20.2\%, 95\% Cl: 19.6-20.8 of the general population perceived the health literacy tasks as difficult or very difficult at the individual item level. On the scale level, the mean rating for i) understanding health information was 3.10, 95\% Cl: 3.09-3.10, and 3.07, 95\% Cl: 3.07-3.08 for ii) engagement with health care providers. Low levels of the two dimensions were associated with low income, low education level, living alone, and to non-Danish ethnicity. Associations with sex and age differed by the specific health literacy dimension.

Conclusion: Estimates on two key dimensions of health literacy in a general population are now available. A substantial proportion of the Danish population perceives difficulties related to understanding health information and engaging with healthcare providers. The study supports previous findings of a socio-economic gradient in health literacy. New insight is provided on the feasibility of measuring health literacy which is of importance for optimising health systems.

Keywords: Health literacy, Health competence, Patient-centred care, Patient participation, Doctor-patient relations, Health information, Socioeconomic factors, Inequality in health, Population survey, Health literacy questionnaire (HLQ)

\footnotetext{
* Correspondence: htm@alm.au.dk

${ }^{1}$ Department of Public Health, Section for Health Promotion and Health

Services, Aarhus University, Aarhus, Denmark

Full list of author information is available at the end of the article
} 


\section{Background}

With the growing complexity of modern health care, the population is confronted with increasing demands to understand and utilize health information. Together with the increasing proportion of people living with chronic conditions, competencies for proactive self-management of health and participation in collaborative care have become key public health agendas [1,2]. The ability to take active part in shared decision making with healthcare providers is important for adherence to treatment, selfmanagement of chronic diseases [2-4], and it is rooted in ethical considerations for patient autonomy $[5,6]$. However, the World Health Organization recently described a 'health decision making paradox' where the increased demands on the individual to make choices for health, are not accompanied by appropriate information and support to enable this to happen [7].

In this context, health literacy has received increased attention as it addresses citizens' motivation and ability to gain access to, understand and use information in ways which promote and maintain good health [8]. The concept has evolved from a focus on functional skills such as reading and understanding health information to a focus in newer definitions on higher order competencies such as critical appraisal of health information and applying it in everyday life and when interacting with the health care system $[9,10]$. The level of skills and competencies required by individuals varies with the contextual demands such as the complexity of the health care system, access to health information or patient education, communication skills of health professionals and their time to convey messages and offer patient support, and the availability of social mobilization or advocacy [11-13]. Also, individuals with more complex conditions face higher management demands.

Health literacy research has its roots in investigations of general adult literacy and health for example the International Adult Literacy Survey and the Adult Literacy and Life Skills Survey [14], and has developed to specifically include health literacy for example in a large health literacy survey from the U.S. using the Health Activities Literacy Scale [15]. Studies have found associations between functional health literacy skills and increased levels of hospitalization, poor adherence to treatment, poorer self-care management of chronic disease, lower participation in screening programs, and higher rates of morbidity and mortality $[16,17]$.

Expanded conceptual frameworks and comprehensive measurement tools for health literacy have recently been developed $[9,11,18-20]$. These deal with both functional skills and higher order health literacy competencies and they use self-report of health literacy related behaviour or self-reported difficulty of health literacy related tasks. In 2012 the European Health Literacy Consortium performed a health literacy survey (HLS-EU) using a selfreport measurement tool in a random sample of approximately 1000 citizens in eight European countries [21]. This study suggested that almost every second citizen has some degree of limited health literacy, as defined by the authors, and limited health literacy was associated with both socio-economic factors and health status [21]. Osborne et al. developed a health literacy conceptual model using consultation approaches grounded in the daily lives of citizens, practitioners and policymakers. From this the multi-dimensional Health Literacy Questionnaire $\left(\mathrm{HLQ}^{\mathrm{m}}\right)$ was developed to cover nine separate dimensions of health literacy from functional levels such as understanding and appraising health information to higher order competencies such as interacting with health care providers and actively managing own health [11]. In our study, health literacy is understood in line with these broad conceptualisations as a multidimensional concept encompassing individual skills and competencies at various levels and these have to be understood and interpreted relatively to the contextual demands.

A socio-economic gradient in health literacy across several socio-economic indicators has been observed in previous studies using both functional tests and selfreported measurement tools, with low health literacy levels observed in population groups with lower levels of education and income, and in ethnic minority groups [21-23]. The increasing demands on individuals to take responsibility for their own health seems to inadvertently increase social inequalities in health as it favours those with high health literacy and education levels [24].

Most research on health literacy is based on small samples, focusses on functional health literacy or specific patient groups $[7,25,26]$. No published studies have focused on health literacy in Denmark or other Nordic countries. There is a need for an expanded understanding of health literacy at the population level to guide health services in their responds to the needs of citizens.

The aim of this study was first to describe the level of two important dimensions of health literacy in the Danish population; 1 ) the ability to understand health information well enough to know what to do, and 2) the ability to actively engage with healthcare providers, and secondly to examine pertinent relationships between socio-economic characteristics with these dimensions of health literacy.

\section{Methods}

\section{Study design and data collection}

A population-based cross sectional study was conducted. Denmark has about 5.5 million inhabitants and is administratively divided into five regions. A sample of 46,354 individuals above 25 years of age was drawn randomly from the Danish Civil Registration System among citizens in the Central Denmark Region, where approximately $22 \%$ 
of the Danish population resides. A total of 29,473 respondents completed and returned the questionnaire giving a response rate of $63.6 \%$.

Data were collected from January to April 2013 through the Central Denmark Region's health survey that was a part of the Danish National Health Survey [27] in 2013. The national survey was sent out to five mutually exclusive random sub-samples in different regions of Denmark. The National Health Survey contains 54 core items on: health, health behaviour, disease, and socio-economic characteristics. In addition, each region can add separate questions. In 2013, ten questions on two dimensions of health literacy were added in The Central Denmark Region's health survey. Participants were recruited by mail with an introduction letter describing the purpose and content of the survey. The wording and lay out of the material was fairly simple but not specifically targeted low literate groups and only provided in Danish. The letter emphasized that participation was voluntary and that data would be treated confidentially. The survey applied a self-administered paper or web-based questionnaire, with a reminder procedure comprising three postal reminders.

\section{Calculation of population weights}

Using the unique personal identification number given to all citizens from the Civil Registration System, both respondents and non-respondents were linked to Danish national registers. We therefore had the opportunity to estimate weights to account for differences in selection probabilities and for differences in response rates for different sub-groups using a model-based calibration approach [28]. Data were weighted to represent the population in the Central Denmark Region. The weights were based on register information on sex, age, municipality of residence, highest completed educational level, income, marital status, country of birth, visit to the general practitioner, hospitalization, occupational status, owner/tenant status, and protection from inquiries during statistical and scientific surveys for all individuals [27].

\section{Assessment of variables \\ Dependent variables}

The health literacy questions were selected from the $\mathrm{HLQ}^{\mathrm{sm}}$ [11]. The HLQ ${ }^{\mathrm{TM}}$ was developed using a validitydriven approach including in depth grounded consultations (workshops and interviews), psychometric analyses, and cognitive interviews. It was initially calibrated among 634 individuals and then confirmed in a replication sample of 405 individuals [11]. The HLQ ${ }^{\mathrm{TM}}$ contains 44 questions across nine independent scales. Given that we were only able to include about 10 items, we selected two scales that strongly reflected core and distinct competencies for participation in the health care process:
Understand health information well enough to know what to do ('Understanding', 5 items), and: ability to actively engage with healthcare providers ('Engagement', 5 items). The ten items covered a range of simple or easy through to more challenging heath literacy-related tasks. For each item, participants indicated their perceived difficulty with the response options; 1 =very difficult, 2 = difficult, $3=$ easy, and $4=$ very easy. Scale scores were used as dependent variables and were calculated for each individual as the mean of item scores for the five items. If responses to more than two items in a scale were missing, the scale score was regarded as missing, if one or two items were missing, the mean of the available items was used as the scale score. We did not set a threshold for low or inadequate levels of health literacy, as this would be an arbitrary choice. Table 1 shows the

\section{Table 1 Health literacy items and interpretation of scale scores for the two health literacy scales, Central Denmark Region 2013}

Understand health information well enough to know what to do ('Understanding')

1a Confidently fill medical forms in the correct way

2a Accurately follow the instructions from...

3a Read and understand written health information

$4 \mathrm{a}$ Read and understand all the information on medication labels

5 a Understand what healthcare providers are asking you to do

High: Individuals with high scores are able to understand all written information (including numerical information) in relation to their health and able to write appropriately on forms where required

Low: Individuals with low scores have problems understanding any written health information or instructions about treatments or medications. Unable to read or write well enough to complete medical forms

Ability to actively engage with healthcare providers ('Engagement')

1b Make sure that healthcare providers understand your problems properly

2b Feel able to discuss your health concerns with a healthcare provider

3b Have good discussions about your health with doctors

$4 \mathrm{~b}$ Discuss things with healthcare providers until you understand all you need to

5b Ask healthcare providers questions to get the health information...

High: Individuals with high scores are proactive about their health and feel in control in relationships with healthcare providers. Are able to seek advice from additional healthcare providers when necessary. They keep going until they get what they want. Empowered

Low: Individuals with low scores are passive in their approach to healthcare, inactive i.e., they do not proactively seek or clarify information and advice and/or service options. They accept information without question. Unable to ask questions to get information or to clarify what they do not understand. They accept what is offered without seeking to ensure that it meets their needs. Feel unable to share concerns. The do not have a sense of agency in interactions with providers

${ }^{1}$ Some HLQ ${ }^{\mathrm{TM}}$ items are truncated. HLQ is protected by copyright and cannot be used without permission of the authors. Full copy of the items is available at hlq@deakin.edu.au. 
questions and interpretation of scale scores. The questions were presented in a random order in the questionnaire. The translation and adaption of the $\mathrm{HLQ}^{\mathrm{m}}$ followed a standardised procedure [29] led by Maindal, Osborne and colleagues to ensure cross-cultural validity. The translation went through five steps including a forward-backward translation, guided by explicit item intent guidance, expert panel discussion, pre-test, and a cognitive testing (manuscript in preparation).

\section{Demographic and socio-economic characteristics}

Demographic and socio-economic factors included: sex, age, ethnicity, education level, personal annual gross income, and cohabitation. Information on sex, age and whether an individual was an immigrant or Danish were collected from national registers to avoid missing data. All other data were self-reported. Immigrants and Danes were identified in registers using Statistics Denmark's definition [30]: Immigrants are foreign-born with neither parent being both Danish citizens and born in Denmark. Descendants are born in Denmark with neither parent both being Danish citizen and born in Denmark. Danes have at least one parent born in Denmark with Danish citizenship. In this study, immigrants and descendants were grouped in the category "immigrants", and we denoted the variable 'Origin'. Mother tongue was grouped as Danish or other language. Using the education nomenclature (ISCED) from Statistics Denmark, educational level was grouped into three categories; low (1-10 years), medium (11-14 years of education), and high ( $>15$ years). Respondents were asked to indicate their approximate gross annual income by marking one of eight income intervals that were chosen based on the general income distribution in the Danish population. For the analysis we grouped the intervals two by two to form the intervals; 0-149,000 DKK, 150,000-374,000 DKK, 375,000699,000 DKK, >700,000 DKK (7.46 DKK equals $1 €)$. Cohabitation included whether an individual lives alone or lives with others (adults and/or children). Finally, we collected information on whether a participant had seen a general practitioner within the last year or not. We assumed that individuals who have not seen a general practitioner in the last year might answer questions about engagement differently, as they had to relate to experiences more than a year before. Further, the number of visits to the general practitioner is known to be associated with socio-economic factors [31]; hence the variable was considered a potential confounder.

\section{Statistical analysis}

As the Danish version of the $\mathrm{HLQ}^{\mathrm{Tm}}$ was used for the first time in a large population in the health survey we investigated basic psychometric properties. For each item, proportions of non-response and population-weighted proportions of respondents in each response category were calculated. The correlation between the two scales was investigated using Pearson's correlation coefficient, and we explored internal consistency using Cronbach's alpha.

The level of perceived difficulty (difficulty level) of each item was calculated as the population-weighted proportion of respondents who perceived the items as difficult or very difficult with $95 \%$ confidence intervals (CI). We calculated the difficulty level of all items for the whole sample and for socio-economic subgroups. Population-weighted multivariate linear regression models were used to assess the associations between socio-economic characteristics and the two dimensions of health literacy on a scale level. Statistical analyses were performed using STATA 13 (StataCorp LP. College Station, TX, USA.).

\section{Ethics}

According to Danish law, approval by the Ethics Committee and written informed consent is not required in questionnaire-based and register-based studies [32]. The provision of information about the survey and its purpose, as well as the voluntary completion and return of the survey by participants constituted implied consent. The study was approved by the Danish Data Protection Agency (j.no: 2007-58-0010) and was undertaken in accordance with the Helsinki Declaration.

\section{Results}

\section{Psychometric properties}

Non-response in the ten health literacy items was low and evenly distributed (between 5.9\% and 7.3\%) (Table 2), suggesting that items were understood and had acceptable content. For all items, all response options were endorsed by some respondents although there were fewer in the extreme 'very difficult' category and many in the "easy" category (Table 2). The population-weighted mean rating for 'Understanding' was 3.10, 95\% CI: 3.093.10, which was marginally higher than for 'Engagement' 3.07, 95\% CI: 3.07-3.08. Median scores on both scales were three (i.e., perceived as easy). The scales correlated positively with Pearson's coefficient $=0.76$. The Cronbach's alpha coefficient indicated high internal consistency of both scales; 'Understanding' $\alpha=0.87$ and 'Engagement' $\alpha=0.91$.

\section{Sample characteristics}

Characteristics of the respondents are presented in Table 3. There were slightly more women than men, $52.5 \%$ versus $47.5 \%$, most were aged $45-64$ years (mean age: 54.8, SD 15.3), and about one in 20 were born outside Denmark or had a non-Danish mother tongue. When comparing the distribution of respondents in the sample with the population-weighted distribution also 
Table 2 Item missing, population-weighted response distribution and difficulty level of items

\begin{tabular}{|c|c|c|c|c|c|c|}
\hline \multirow{3}{*}{ Items ${ }^{2}$} & \multirow[t]{2}{*}{ Item missing } & \multicolumn{4}{|c|}{ Population-weighted ${ }^{1}$ proportion in each response category } & \multirow{2}{*}{$\begin{array}{l}\text { Population-weighted proportion of population } \\
\text { reporting difficult or very difficult across items }\end{array}$} \\
\hline & & Very difficult & Difficult & Easy & Very easy & \\
\hline & $\%$ & $\%$ & $\%$ & $\%$ & $\%$ & $\%(95 \% \mathrm{Cl})$ \\
\hline \multicolumn{7}{|c|}{ Understanding $^{2}$} \\
\hline $1 \mathrm{a}$ & 6.9 & 2.6 & 13.4 & 57.2 & 26.7 & $16.0(15.5-16.6)$ \\
\hline $2 a$ & 7.3 & 1.4 & 13.1 & 61.3 & 24.2 & $14.5(14.0-15.0)$ \\
\hline $3 a$ & 6.7 & 2.0 & 10.8 & 58.3 & 28.9 & $12.8(12.4-13.3)$ \\
\hline $4 a$ & 6.4 & 3.2 & 17.0 & 56.0 & 23.8 & $20.2(19.6-20.8)$ \\
\hline $5 a$ & 7.0 & 1.0 & 7.8 & 64.7 & 26.6 & $8.8(8.4-9.2)$ \\
\hline \multicolumn{7}{|c|}{ Engagement $^{2}$} \\
\hline $1 b$ & 6.8 & 2.5 & 15.8 & 57.8 & 24.0 & $18.3(17.7-18.8)$ \\
\hline $2 b$ & 6.5 & 1.7 & 12.8 & 57.4 & 28.2 & $14.5(14.0-15.0)$ \\
\hline $3 b$ & 5.9 & 2.2 & 14.4 & 56.2 & 27.3 & $16.6(16.1-17.1)$ \\
\hline $4 b$ & 7.3 & 2.0 & 16.3 & 56.9 & 24.8 & $18.3(17.8-18.9)$ \\
\hline $5 b$ & 7.0 & 1.6 & 13.7 & 59.2 & 25.5 & $15.3(14.8-15.8)$ \\
\hline
\end{tabular}

$\mathrm{n}=29,473$, Central Denmark Region 2013.

${ }^{1}$ The sample is weighted based on register data to represent the population of the Central Denmark Region 2013.

${ }^{2}$ See Table 1 for description of items and scales.

presented in Table 3, it appears that some groups are less represented among the respondents than in the general population, for example the youngest age groups, where the percentage is $27.5 \%$ in the sample and $36.6 \%$ in the weighted population. Missing data on socioeconomic characteristics was low; $5.3 \%$ for income and zero for register-based data (sex, age and origin).

\section{Difficulty in undertaking core health literacy tasks}

The population-weighted difficulty level of each item is presented in Table 2. The items that were rated least and most difficult were both found in the scale 'Understanding'; the item with the highest difficulty level was: 'Read and understand all the information on medication labels' (4a) 20.2\%, 95\% CI: 19.6-20.8, and the easiest task was 'Understand what healthcare providers are asking you to do' (5a) 8.8\%, 95\% CI: 8.4-9.2. The items perceived most difficult in the 'Engagement' scale were: 'Make sure that health care providers understand your problems properly' (1b) 18.3\%, 95\% CI: 17.7-18.8 and 'Discuss things with healthcare providers until you understand all you need to' (4b) 18.3\%, 95\% CI: 17.818.9. The task perceived most easy in the 'Engagement' scale was: 'Feel able to discuss your health concerns with a healthcare provider' (2b) 14.5\%, 95\% CI: 14.0-15.0.

\section{Core health literacy tasks by socio-economic characteristics}

Table 3 and Figure 1 show population-weighted crude estimates of difficulty levels by socio-economic characteristic for each item. Overall, the difficulty level of the ten items varied between subgroups in the population. Regarding sex, women reported somewhat higher difficulty than men in all items in the 'Engagement' scale, especially in items $1 b, 2 b$ and $3 b$ that reflect the quality of the communication with healthcare providers. In the 'Understanding' scale, the levels were quite similar, except for item $4 \mathrm{a}$, where men perceived more difficulty than women in understanding information on medication labels.

For age, the difficulty level was rather similar across the ages of 25-64 years. The 65-84 year olds perceived more difficulty than the younger age groups regarding the functional skills; filling out medical forms (1a) and reading and understanding health information (3a), but perceived less difficulty than the younger age groups regarding items that reflected a direct contact with healthcare providers (1b-4b), as well as following instruction from health care providers (2a). Individuals aged 85 years or older perceived most items as being more difficult than the younger age groups. Among the oldest individuals, the highest difficulty levels were found for the functional skills reflected in items 1a, 3a, and 4a, where about one third perceived the items as difficult or very difficult. However, also discussing things with health care providers (4b) was rated particularly difficult by the oldest age group.

Both immigrants and the group with non-Danish mother tongue perceived more difficulty than Danes in all items. The largest difference was in reading and understanding health information (3a) where immigrants were more than twice as likely to report difficulties. 
Table 3 Participant characteristics and population-weighted difficulty level by socio-economic characteristics, Central Denmark Region 2013

\begin{tabular}{|c|c|c|c|c|c|c|c|c|c|c|c|c|c|}
\hline \multirow[b]{4}{*}{ Characteristics } & \multirow{2}{*}{\multicolumn{3}{|c|}{ Distribution in sample }} & \multicolumn{10}{|c|}{$\begin{array}{c}\text { Population-weighted }{ }^{1} \text { proportion of population reporting difficult } \\
\text { or very difficult across items }{ }^{2}\end{array}$} \\
\hline & & & & \multicolumn{5}{|c|}{ Understanding $^{2}$} & \multicolumn{5}{|c|}{ Engagement $^{2}$} \\
\hline & \multicolumn{3}{|c|}{ Total $(n=29,473)$} & $1 \mathrm{a}$ & $2 a$ & $3 a$ & $4 a$ & $5 a$ & $1 b$ & $2 b$ & $3 b$ & $4 b$ & $5 b$ \\
\hline & $\mathbf{N}$ & $\%$ & Weighted $^{1} \%$ & \multicolumn{10}{|c|}{ (\%) } \\
\hline \multicolumn{14}{|l|}{ Sex } \\
\hline Female & 15,459 & 52.5 & 50.6 & 17.4 & 14.6 & 12.6 & 18.3 & 9.1 & 21.0 & 17.0 & 19.2 & 19.4 & 16.2 \\
\hline Male & 14,014 & 47.5 & 49.4 & 14.7 & 14.4 & 13.0 & 22.2 & 8.5 & 15.6 & 12.0 & 13.9 & 17.3 & 14.3 \\
\hline Missing & 0 & 0.0 & 0.0 & & & & & & & & & & \\
\hline \multicolumn{14}{|l|}{ Age } \\
\hline $25-44$ years & 8,102 & 27.5 & 36.6 & 12.8 & 14.7 & 10.2 & 17.3 & 8.2 & 19.6 & 16.1 & 18.6 & 19.2 & 15.2 \\
\hline 45-64 years & 12,701 & 43.1 & 38.6 & 15.5 & 15.5 & 11.8 & 20.4 & 8.5 & 18.3 & 14.5 & 17.2 & 18.1 & 15.3 \\
\hline 65-84 years & 8,046 & 27.3 & 22.4 & 20.7 & 11.8 & 17.6 & 23.4 & 9.4 & 15.4 & 11.1 & 11.5 & 16.3 & 14.6 \\
\hline$=>85$ years & 624 & 2.1 & 2.4 & 41.9 & 19.3 & 32.9 & 36.9 & 19.8 & 24.1 & 20.6 & 21.3 & 29.1 & 25.7 \\
\hline Missing & 0 & 0.0 & 0.0 & & & & & & & & & & \\
\hline \multicolumn{14}{|l|}{ Origin } \\
\hline Danish & 28,400 & 96.4 & 93.6 & 15.6 & 14.2 & 11.9 & 19.7 & 8.2 & 17.7 & 13.9 & 16.2 & 17.8 & 14.6 \\
\hline Immigrant & 1,073 & 3.6 & 6.4 & 22.6 & 19.2 & 26.1 & 27.2 & 17.3 & 27.8 & 23.5 & 23.0 & 26.5 & 25.2 \\
\hline Missing & 0 & 0.0 & 0.0 & & & & & & & & & & \\
\hline \multicolumn{14}{|l|}{ Mother tongue } \\
\hline Danish & 27,565 & 93.5 & 90.3 & 15.4 & 14.0 & 11.8 & 19.6 & 8.1 & 17.4 & 13.7 & 16.0 & 17.7 & 14.5 \\
\hline Other language & 1,441 & 4.9 & 8.0 & 22.1 & 18.5 & 24.0 & 26.6 & 14.9 & 26.8 & 22.0 & 21.6 & 24.6 & 23.3 \\
\hline Missing & 467 & 1.6 & 1.8 & & & & & & & & & & \\
\hline \multicolumn{14}{|l|}{ Cohabitation } \\
\hline Living with others & 23,755 & 80.6 & 75.9 & 14.2 & 13.7 & 11.4 & 19.2 & 8.0 & 17.1 & 13.6 & 16.2 & 17.3 & 14.2 \\
\hline Living alone & 5,078 & 17.2 & 21.9 & 22.6 & 17.6 & 17.9 & 23.7 & 11.7 & 22.6 & 17.9 & 18.2 & 22.1 & 19.1 \\
\hline Missing & 640 & 2.2 & 2.2 & & & & & & & & & & \\
\hline \multicolumn{14}{|l|}{ Education level } \\
\hline Low & 5,507 & 18.7 & 18.0 & 33.9 & 20.9 & 28.6 & 31.9 & 17.7 & 25.2 & 20.9 & 20.3 & 26.9 & 23.4 \\
\hline Medium & 14,718 & 49.9 & 48.4 & 14.6 & 14.7 & 11.7 & 20.8 & 8.0 & 18.0 & 13.8 & 16.2 & 17.8 & 14.8 \\
\hline High & 8,319 & 28.2 & 30.2 & 7.5 & 10.3 & 4.6 & 12.0 & 4.4 & 14.4 & 11.4 & 15.1 & 14.2 & 10.9 \\
\hline Missing & 929 & 3.2 & 3.5 & & & & & & & & & & \\
\hline \multicolumn{14}{|l|}{ Income (DKK) ${ }^{3}$} \\
\hline $0-149,000$ & 6,302 & 21.4 & 22.2 & 24.5 & 17.7 & 21.7 & 26.8 & 14.2 & 23.8 & 19.8 & 19.3 & 23.4 & 21.2 \\
\hline $150,000-374,000$ & 14,417 & 48.9 & 48.4 & 15.6 & 14.4 & 11.7 & 19.4 & 8.0 & 18.9 & 14.4 & 17.1 & 18.6 & 15.0 \\
\hline $375,000-699,000$ & 6,132 & 20.8 & 20.4 & 7.6 & 11.8 & 5.6 & 15.5 & 4.9 & 11.8 & 9.3 & 13.3 & 13.0 & 10.3 \\
\hline$>700,000$ & 1,054 & 3.6 & 3.6 & 4.6 & 11.7 & 3.6 & 12.8 & 3.7 & 10.2 & 8.0 & 12.3 & 9.8 & 7.9 \\
\hline Missing & 1,568 & 5.3 & 5.4 & & & & & & & & & & \\
\hline \multicolumn{14}{|l|}{ GP visit last year ${ }^{4}$} \\
\hline Yes & 22,360 & 75.9 & 74.8 & 17.1 & 15.5 & 13.5 & 20.9 & 9.1 & 20.0 & 15.1 & 17.0 & 19.1 & 15.9 \\
\hline No & 6,663 & 22.6 & 23.6 & 12.5 & 11.5 & 10.6 & 17.9 & 7.7 & 12.7 & 12.5 & 15.3 & 16.1 & 13.4 \\
\hline Missing & 450 & 1.5 & 1.6 & & & & & & & & & & \\
\hline
\end{tabular}

${ }^{1}$ The sample is weighted based on register data to represent the population of the Central Denmark Region 2013.

${ }^{2}$ See Table 1 for description of items and scales.

${ }^{3}$ Personal annual gross income. Exchange rate: 7.46 DKK/1 $€$

${ }^{4} \mathrm{At}$ least one visit to the general practitioner within the last year. 

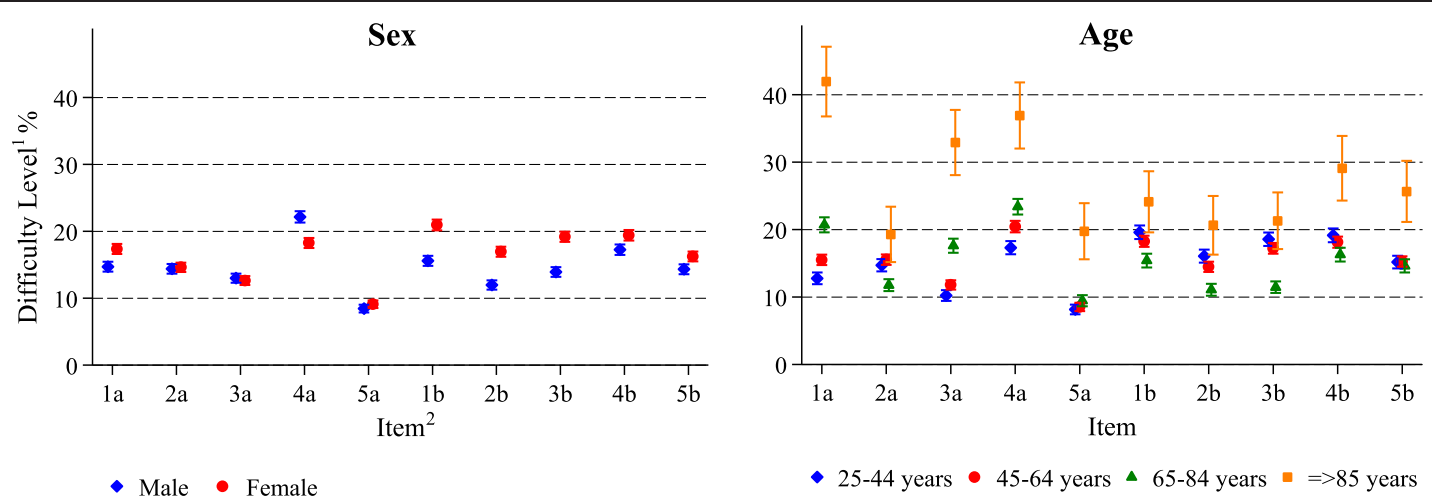

- 25-44 years $\bullet$ 45-64 years $\wedge$ 65-84 years $"=>85$ years
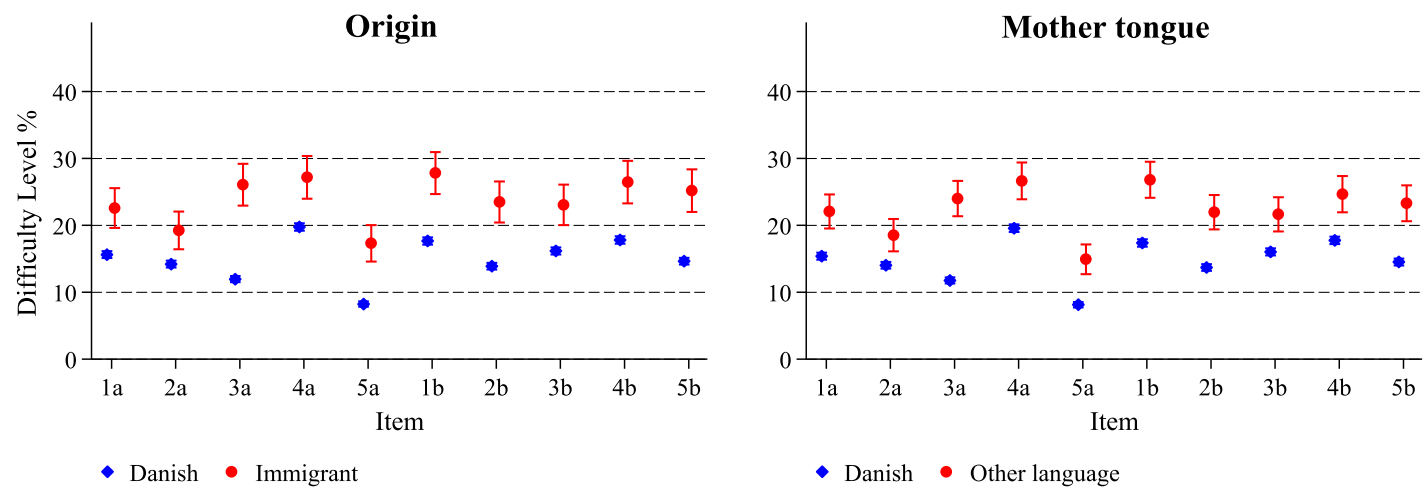

- Danish - Other language
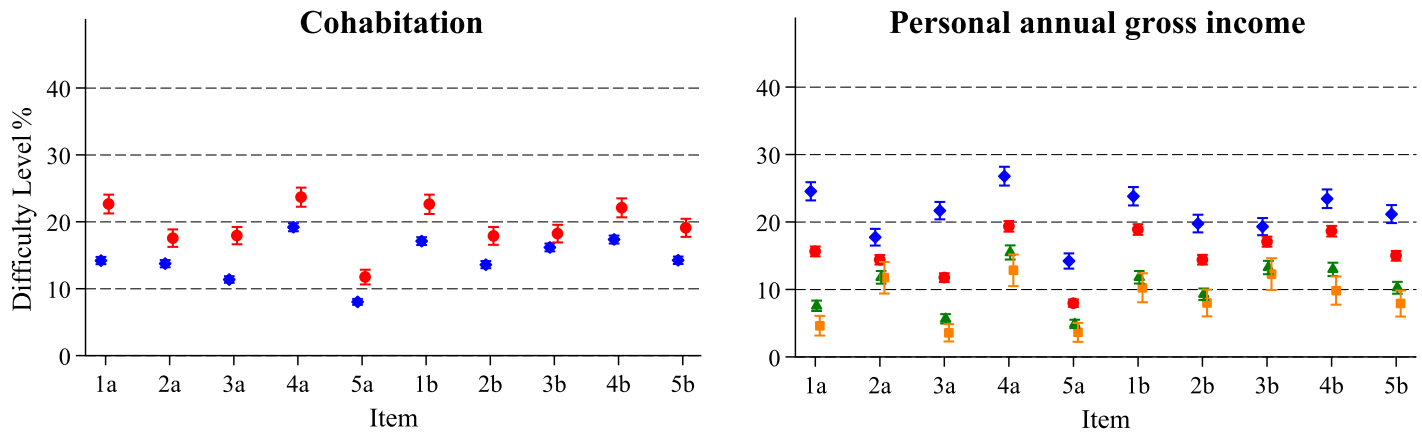

- Living with others $\quad$ Living alone

- $0-149 * \bullet 150-374 * \Delta 375-699 *->700 * * 10^{\wedge} 3 \mathrm{DKK}$
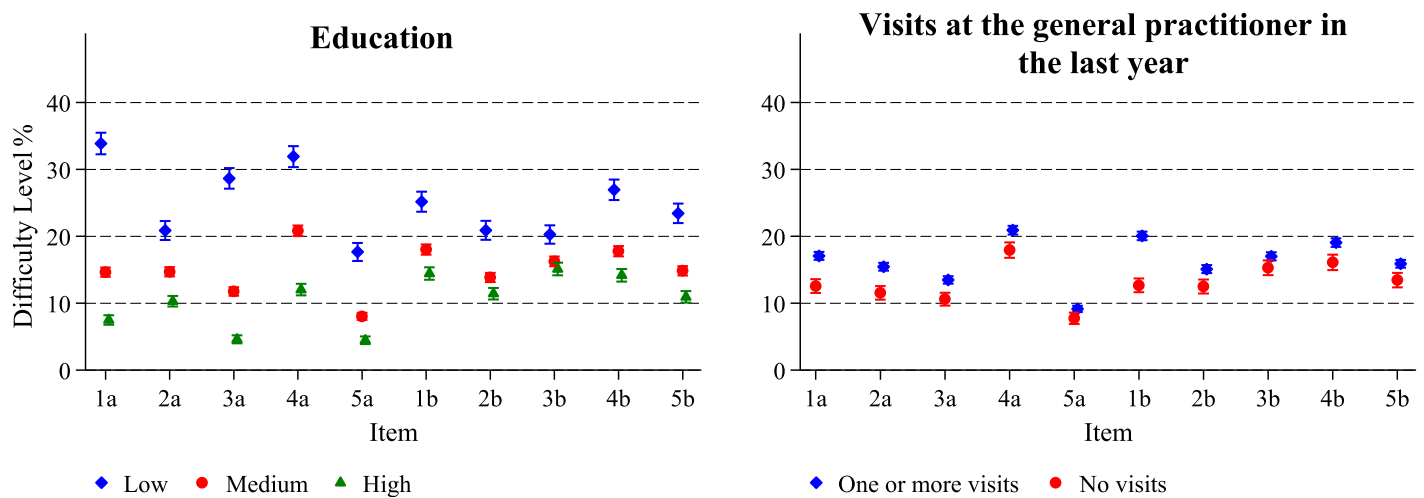

Figure 1 Population-weighted difficulty level of each item by socio-economic characteristic, Central Denmark Region 2013. ${ }^{1}$ Difficulty level is calculated as the population-weighted proportion reporting difficult or very difficult. The sample is weighted based on register data to represent the population of the Central Denmark Region 2013. ${ }^{2}$ See Table 1 for description of items and scales. 
People who live alone also reported more difficulties across all items than those who live with others. Regarding income and education, there was a clear pattern of an increasing difficulty level with less education or lower income across all items. The most striking pattern was a more than four-fold difference in difficulty level between the lowest and highest education group in the tasks of filling medical forms correctly (1a) and of reading and understanding health information (3a). Those who had visited a general practitioner within the last year perceived all items as slightly more difficult than those, who had not.

\section{Association between socio-economic factors and two health literacy scales 'Understanding' and 'Engagement'}

Table 4 shows results of the linear regression analyses with scale means of the two health literacy dimensions as dependent variables. In Model 1, for each scale, the univariate associations are reported. Model 2 then reports the results when including all the socio-economic factors in the table and the possible confounder 'visit at the general practitioner in the last year'.

The significance of sex varied across scales. For 'Understanding', men compared with women had a lower mean level in the adjusted model but for 'Engagement', men had a higher mean score than women in the adjusted model.

Regarding age, individuals aged 65-84 years had higher mean scores (i.e. perceived less difficulties) compared with the reference group (25-44 years) in both scales, when adjusting for all the selected covariates. The adjusted results for the age group 45-64 years differed between the scales. For 'Understanding', age between 45-64 years was associated with a lower mean level compared with the 25-44 year olds, but for 'Engagement', no difference was observed between this age group and the reference group. The same pattern was seen for the oldest age group, with lower mean levels in 'Understanding' but no difference in 'Engagement' compared with the youngest.

Table 4 Associations between health literacy scales and socio-economic characteristics

\begin{tabular}{|c|c|c|c|c|c|c|c|c|}
\hline & \multicolumn{4}{|c|}{ Understanding $^{1}$} & \multicolumn{4}{|c|}{ Engagement $^{1}$} \\
\hline & \multicolumn{2}{|c|}{ Univariate $^{2}$} & \multicolumn{2}{|c|}{ Adjusted $^{3}$} & \multicolumn{2}{|c|}{ Univariate $^{2}$} & \multicolumn{2}{|c|}{ Adjusted $^{3}$} \\
\hline & $\beta$ & $(95 \% \mathrm{Cl})$ & B & $(95 \% \mathrm{Cl})$ & B & $(95 \% \mathrm{Cl})$ & $\beta$ & $(95 \% \mathrm{Cl})$ \\
\hline \multicolumn{9}{|c|}{ Sex (reference group: female) } \\
\hline Male & -0.01 & $(-0.03,0.00)$ & -0.04 & $(-0.06,-0.03)$ & 0.06 & $(0.04,0.08)$ & 0.03 & $(0.01,0.05)$ \\
\hline \multicolumn{9}{|c|}{ Age (reference group: age $25-44$ ) } \\
\hline $45-64$ & -0.06 & $(-0.08,-0.04)$ & -0.03 & $(-0.04,-0.01)$ & 0.00 & $(-0.02,0.02)$ & 0.02 & $(-0.00,0.04)$ \\
\hline $65-84$ & -0.08 & $(-0.10,-0.06)$ & 0.07 & $(0.04,0.09)$ & 0.06 & $(0.04,0.08)$ & 0.18 & $(0.16,0.21)$ \\
\hline$=>85$ & -0.34 & $(-0.42,-0.27)$ & -0.13 & $(-0.22,-0.05)$ & -0.14 & $(-0.21,-0.06)$ & 0.07 & $(-0.02,0.15)$ \\
\hline \multicolumn{9}{|c|}{ Origin (reference group: Danish) } \\
\hline Immigrant & -0.14 & $(-0.19,-0.09)$ & -0.09 & $(-0.17,-0.02)$ & -0.18 & $(-0.22,-0.13)$ & -0.09 & $(-0.17,-0.01)$ \\
\hline \multicolumn{9}{|c|}{ Mother tongue (reference group: Danish) } \\
\hline Other language & -0.12 & $(-0.16,-0.08)$ & -0.02 & $(-0.08,0.04)$ & -0.15 & $(-0.19,-0.11)$ & -0.03 & $(-0.10,0.03)$ \\
\hline \multicolumn{9}{|c|}{ Living situation (reference group: With others) } \\
\hline Alone & -0.11 & $(-0.13,-0.09)$ & -0.05 & $(-0.07,-0.03)$ & -0.08 & $(-0.11,-0.06)$ & -0.06 & $(-0.09,-0.04)$ \\
\hline \multicolumn{9}{|c|}{ Education level (reference group: High) } \\
\hline Medium & -0.21 & $(-0.23,-0.20)$ & -0.19 & $(-0.21,-0.18)$ & -0.13 & $(-0.15,-0.11)$ & -0.13 & $(-0.15,-0.11)$ \\
\hline Low & -0.44 & $(-0.47,-0.42)$ & -0.38 & $(-0.41,-0.35)$ & -0.26 & $(-0.29,-0.24)$ & -0.25 & $(-0.28,-0.22)$ \\
\hline \multicolumn{9}{|c|}{ Income $(\text { DKK) })^{4}$ (reference group: $>700,000$ ) } \\
\hline $0-149,000$ & -0.37 & $(-0.42,-0.33)$ & -0.25 & $(-0.30,-0.21)$ & -0.32 & $(-0.37,-0.28)$ & -0.26 & $(-0.31,-0.21)$ \\
\hline $150,000-374,000$ & -0.26 & $(-0.30,-0.22)$ & -0.18 & $(-0.22,-0.14)$ & -0.25 & $(-0.29,-0.21)$ & -0.18 & $(-0.23,-0.14)$ \\
\hline $375,000-699,000$ & -0.13 & $(-0.17,-0.09)$ & -0.10 & $(-0.14,-0.06)$ & -0.12 & $(-0.16,-0.08)$ & -0.09 & $(-0.13,-0.05)$ \\
\hline
\end{tabular}

Mean scale scores allowing for two missing responses are used as dependent variable in the regression models; statistically significant differences are printed in bold $(p<0.05)$. The sample is weighted based on register data to represent the population of the Central Denmark Region 2013.

${ }^{1}$ See Table 1 for description of scales.

2Unadjusted models of the association between each socio-economic characteristic for the scales 'Understanding' and 'Engagement'. Number of individuals varies for the different models due to missing data in the respective socio-economic factor. 'Understanding': $n=26,557-27,512$. 'Engagement': $n=26,589-27,549$.

${ }^{3}$ Model adjusted for all the socio-economic characteristics in table and for the confounder 'visit at the general practitioner in the last year'. 'Understanding': $\mathrm{n}=25,486$, 'Engagement': $n=25,514$.

${ }^{4}$ Personal annual gross income. Exchange rate: $7.46 \mathrm{DKK} / 1 €$.

Unadjusted and adjusted models, Central Denmark Region 2013. 
In the unadjusted models both origin and mother tongue were associated with the two health literacy scales, but in the adjusted models including both measures of ethnicity, only origin remained significant, with lower mean levels among immigrants. Also, when adjusting for other factors, individuals who were living alone had lower scores compared with those who live with others.

The largest differences between subgroups were found for income and education level. For both scales, lower income and education level were associated with lower mean scores, when adjusting for other socio-economic factors.

\section{Discussion}

Danish estimates of two key dimensions of health literacy are now available showing that $10-20 \%$ of the Danish population perceive difficulties in tasks related to: 'Ability to understand health information well enough to know what to do' and 'Ability to actively engage with healthcare providers'. In people from lower socioeconomic groups, those with non-Danish ethnicity, and older people, the proportion reporting difficulties is as high as 20 to $40 \%$. All investigated socio-economic characteristics including sex, age, the two measures of ethnicity, education, income, and cohabitation were independently associated with the two investigated health literacy dimensions.

Given that this study was undertaken in country with a universal healthcare system with multiple policies promoting patient empowerment, patient centred care, user involvement in health planning, and health communication it is concerning that a significant part of the population is reporting difficulties in functional health literacy tasks and in engaging with healthcare providers. Especially considering that those with very poor literacy skills may have been unable to participate due to the selfadministered written format. Our results may reflect the increasing complexity of medical treatments, in combination with a healthcare system that constraints resources such as time, staff and money. Furthermore, the OECD Skills Survey from 2012 found that one in six individuals in Denmark has a poor literacy level [33] and Denmark ranged below otherwise comparable Nordic countries as Norway and Sweden [34]. This may add to the explanation to our findings, especially for the functional scale.

The validation study of the HLQ ${ }^{\mathrm{TM}}[11]$, undertaken in a healthcare system similar to the Danish with universal access and primary care as entry point, provide data on the percentage of respondents rating items as difficult or very difficult. In the scale 'Understanding' the percentage varied between $8-16 \%$, compared to $8-20 \%$ in our study, and in the scale 'Engagement' it varied between 15-24\% compared to $15-24 \%$. In both studies, the most difficult item in the scale 'Understanding' was: Read and understand all the information on medication labels (4a) and in the scale 'Engagement': Discuss things with healthcare providers until you understand all you need to (4b). However, care should be taken comparing the two studies directly, as we did not use the most extreme response category 'Cannot do'. In a comparison of eight different European countries, the recent HLS-EU [21] study classified $29 \%$ to $62 \%$ of the population as having inadequate or limited health literacy depending on the specific country. Other studies from the U.S. and Australia have found that the prevalence of low or suboptimal health literacy, as categorized by the authors, ranged from as low as $7 \%$ to a high of $60 \%[15,22,23]$.

The socio-economic gradient found in our study is clear and previous studies of either self-reported difficulty or abilities measured by functional tests have also documented such gradients [21-23,35]. In Denmark, 98\% of the population have an assigned general practitioner. These follow common rules, medical recommendations, and budgetary agreements. Furthermore, tertiary care is almost exclusively carried out by public hospitals. Therefore, it is likely that the observed social gradient primarily reflects actual differences in individual competencies rather than differences in for example quality of treatment and patient support. It is important to note that the response rate to the survey was $63.6 \%$ and non-respondents are more likely to comprise the more socially deprived groups. Nonetheless, the social gradient is clear and future studies would benefit from focused research protocols that include and oversample the 'hard to reach' groups.

Previous studies have also found that education is strongly linked to functional health literacy skills and the ability to act upon health information $[9,10]$, and people with lower education have been found to have lower health literacy in comparison to people with higher education [7,22,23]. A recent study suggested that health literacy is a likely mediator for the association between education and health [36]. However, programs have shown that health literacy competencies can be achieved even in individuals with low literacy [10]. Income is the social status indicator that most directly measures material living standards, but is interlinked with other indicators such as employment status, job type, access to health promoting services, self-esteem, and to relative social standing in society [37]. Both education and income being significant in the same model shows that health literacy is tied to more complex social structures than can be described by education alone, which is in line with the complex frameworks for health literacy $[9,38]$. Among ethnic minority groups, language can be a barrier to communication with health care providers and make obtaining and processing oral and written health information difficult and thereby lead to low 
health literacy [39]. We found that, after adjustment for mother tongue, origin remained associated with both 'Engagement' and 'Understanding'. This indicates that other aspects such as perception of health and disease, self-efficacy and other personal resources, or quality of care may determine ethnic differences in health literacy. Living alone was also associated with a lower level of health literacy. Health literacy has been found to be distributed through family and social networks [40], where resources such as knowledge, support for health decision making and communication with health care professionals are passed on between close relations. Thereby, low health literacy may well be compensated by support through family and friends [11].

Older age has often been associated with lower health literacy levels [21,23], but this study found that compared to the 25-45 year olds, the age group 45-65 years reported less difficulties in 'Engagement' and 'Understanding'. In Denmark all citizens are affiliated with a general practitioner in their geographical region [41]. Therefore an established relationship with their general practitioner, together with longer experience in navigating the health care system, may have strengthened the 45-65 year olds capabilities. For the scale 'Understanding', age 85 years or older was significantly associated with lower health literacy levels, but this was not found in the 'Engagement' scale. Functional health literacy might decrease with decreasing cognitive abilities of the elderly, and older individuals might face increasingly complex health problems. However, this may not affect their perception of quality of the dialogue with healthcare providers. It is also possible that people in this older generation do not expect to be a participating partner with the health professional and therefore perceive the tasks in the interaction scale as less problematic. Regarding sex, previous studies have found either no differences, or that one of the sexes is more likely to have low health literacy $[21,23,42]$. This study found that the influence of sex was modest and depended on the specific scale. The HLS-EU study found that women compared to men have slightly higher health literacy levels [21].

Our results indicate a need for population level interventions at two levels; optimizing the health system and promoting health literacy in the general population, for example through school-based interventions. The two dimensions of health literacy reflect an individual's ability to utilize health information actively in decision making about health, and the ability to be proactive and feeling in control in relationships with healthcare providers. Our analysis of specific items guides the improvement of the health system. For example, the perceived difficulty of discussing problems and feeling understood by the health care provider may well reflect a lack of time in the consultation, or lack of communication training of the health care providers. In the functional dimension, our results for example indicate a need for improving the information on medication labels.

\section{Strength and limitations}

This study is the largest health literacy study of individual respondents to date, using a robust measure of health literacy, and employing a sampling frame that was population-based and weighted. The large sample allowed us to perform a nuanced investigation at both the scale and item level, and to investigate the two health literacy dimensions across several socio-economic factors. Furthermore, applying population weights compensated for non-response which made our results representative to the general population. While the cross sectional design of this study limits conclusions about causal associations, it identified vulnerable population groups and provided new insights into plausible individual, healthcare and public health interventions that are required. These population data can be used as norms or benchmarks and can inform future surveys in countries with comparable health systems. There is a need for follow-up studies to develop a better understanding the causal pathway between social status and health literacy as well as for studies that also include analysis of contextual factors. Experimental studies are also needed to identify appropriate interventions.

We used health literacy questions that were developed and translated using a validity driven approach, and they worked well when applied in the population health survey. As opposed to the original HLQ ${ }^{\mathrm{Tm}}$ the research team together with the $\mathrm{HLQ}^{\mathrm{m}}$ authors chose not to include the extreme response category 'cannot do'. The very low frequency of endorsement of the 'very difficult' response option at $1-3 \%$ across items (Table 2) indicates that the fifth response option would have been redundant and would not have improved the sensitivity of the survey, nor the conclusion of the results. The category 'easy' was used very often. This might impair the possibility to detect differences between groups of people with high health literacy if the questionnaire is used in smaller samples. Therefore, there may be a need to revise response categories in future studies. The self-reported difficulty format worked well in this large survey and gave a nuanced picture of the challenges the public perceive within the two dimensions. It is important to note that the self-report format is not intended to capture actual skills, but instead reflects what the individual experiences in relation to the health literacy demands in their environment, given whatever level of skills they may have. Some studies have shown that tests of functional skills correlate well with self-reported measures [21,43], but future studies may be strengthened by including the full range of robust psychometric health literacy scales 
in the HLQ ${ }^{\mathrm{Tm}}$ alongside functional tests. Nonetheless, in the current study we have provided fine-grained indicators of two dimensions of health literacy that provide specific and important data of immediate relevance for primary care health services planning and priorities.

Even though the study applied population weights, a slight overestimate of the levels of the health literacy dimensions is possible, as those with very low literacy skills or poor Danish language skills may not have been able to participate. In fact, ability and motivation to fill out at health survey can in itself be viewed as a health literacy competence, and applying a self-administered health literacy survey may therefore exclude the most vulnerable groups. Minimal bias due to missing data on socio-economic characteristics is expected, as this was generally low.

The study was limited to two key dimensions of health literacy. It is possible that more striking elements of health literacy strengths and weaknesses may emerge through further investigation of the full range of health literacy concepts present within the $\mathrm{HLQ}^{\mathrm{m}}$. It is critical that future studies investigate the relationship between health literacy and health outcomes.

\section{Conclusions}

In Denmark, around 10 to $20 \%$ of the general population perceive difficulties in key health literacy dimensions; ability to understand information well enough to know what to do, and ability to actively engage with healthcare providers. Perceived health literacy difficulties are markedly higher in people above 85 years, people with lower income and education level, people who live alone and in people with non-Danish ethnicity. This study provides new and fine-grained information on the health literacy needs across the general population. In particular, the findings reveal a need for population level interventions especially in support of vulnerable and disadvantaged groups and for health policy responses to optimise the health system in Denmark and similar countries.

\section{Abbreviations}

Cl: Confidence Intervals; DKK: Danish Kroner; HLQ'M: Health Literacy

Questionnaire; HLS-EU: European Health Literacy Survey; ISCED: International Standard Classification of Education; SD: Standard Deviation.

\section{Competing interests}

The study was supported by the pharmaceutical company MSD Denmark Funding was given as an unrestricted research grant. The authors declare that they have no competing interest.

\section{Authors' contributions}

HTM and $A B$ conceived the study question. AB, HTM and KF participated in the design. KF was responsible for the collected data. $A B$ analysed and interpreted the data together with $\mathrm{HTM}, \mathrm{KF}$ and $\mathrm{RHO}$. AB, RHO and HTM drafted the manuscript. All authors participated in interpretation of the results, and contributed to the final writing of the paper. All authors critically revised the manuscript for important intellectual content and approved the final version.

\section{Acknowledgements}

We would like to thank The Public Health and Quality Improvement, Central Denmark Region, for the collection of data, data analysis and data management, Lars Kayser and Ole Noergaard, who also participated in the group behind the Danish translation of the HLQ ${ }^{\text {TM; }}$, bio-statisticians Morten Frydenberg and Gerald Elsworth, and not least the citizens for their time and consideration for participating in the health survey.

\section{Author details}

'Department of Public Health, Section for Health Promotion and Health Services, Aarhus University, Aarhus, Denmark. ${ }^{2}$ Public Health and Quality Improvement, Central Denmark Region, Aarhus, Denmark. ${ }^{3}$ Public Health Innovation, Population Health Strategic Research Centre, School of Health and Social Development, Deakin University, Melbourne, Australia.

Received: 13 January 2014 Accepted: 2 October 2014

Published: 22 October 2014

\section{References}

1. Bodenheimer T, Lorig K, Holman H, Grumbach K: Patient self-management of chronic disease in primary care. JAMA 2002, 288(19):2469-2475.

2. Jordan JE, Osborne RH: Chronic disease self-management education programs: challenges ahead. Med J Aust 2007, 186(2):84-87.

3. Coleman K, Austin BT, Brach C, Wagner EH: Evidence on the chronic care model in the new millennium. Health Aff (Millwood) 2009, 28(1):75-85.

4. Joosten EA, DeFuentes-Merillas $L$, de Weert GH, Sensky $T$, van der Staak $C P$, de Jong CA: Systematic review of the effects of shared decision-making on patient satisfaction, treatment adherence and health status. Psychother Psychosom 2008, 77(4):219-226.

5. Charles $C$, Whelan T, Gafni A: What do we mean by partnership in making decisions about treatment? BMJ 1999, 319(7212):780-782.

6. Appelbaum PS: Assessment of Patients' competence to consent to treatment. N Engl J Med 2007, 357(18):1834-1840.

7. Kickbusch I, Pelikan JM, Apfel F, Tsouros AD: Health Literacy, the Solid Facts. World Health Organization, Regional Office for Europe; 2013 [http://www. euro.who.int/_data/assets/pdf_file/0008/190655/e96854.pdf]

8. Nutbeam D: Health promotion glossary. Health Promot 1999, 12(4):349-364.

9. Sorensen K, Van den Broucke S, Fullam J, Doyle G, Pelikan J, Slonska Z, Brand H, (HLS-EU) Consortium Health Literacy Project European: Health literacy and public health: a systematic review and integration of definitions and models. BMC Public Health 2012, 12:80.

10. Nutbeam D: Health literacy as a public health goal: a challenge for contemporary health education and communication strategies into the 21st century. Health Promot Int 2006, 15(3):259-267.

11. Osborne RH, Batterham RW, Elsworth GR, Hawkins M, Buchbinder R: The grounded psychometric development and initial validation of the Health Literacy Questionnaire (HLQ). BMC Public Health 2013, 13:658.

12. Volandes $A E$, Paasche-Orlow MK: Health literacy, health inequality and a just healthcare system. Am J Bioeth 2007, 7(11):5-10.

13. Rudd RE: Needed action in health literacy. J Health Psychol 2013, 18(8):1004-1010.

14. Statistics Canada, OECD: Learning a Living: First Results from the Adult Literacy and Life Skills Survey. Minister of Industry, Canada and OECD; 2005:30 [http://www.oecd.org/edu/innovation-education/34867438.pdf]

15. Rudd RE: Health literacy skills of U.S. adults. Am J Health Behav 2007, 31(Suppl 1):S8-S18.

16. Berkman ND, Sheridan SL, Donahue KE, Halpern DJ, Crotty K: Low health literacy and health outcomes: an updated systematic review. Ann Intern Med 2011, 155(2):97-107.

17. Schillinger D, Grumbach K, Piette J, Wang F, Osmond D, Daher C, Palacios J, Sullivan GD, Bindman AB: Association of health literacy with diabetes outcomes. JAMA 2002, 288(4):475-482.

18. Chinn D, McCarthy C: All Aspects of Health Literacy Scale (AAHLS): developing a tool to measure functional, communicative and critical health literacy in primary healthcare settings. Patient Educ Couns 2013, 90(2):247-253.

19. Ishikawa $H$, Takeuchi T, Yano E: Measuring functional, communicative, and critical health literacy among diabetic patients. Diabetes Care 2008, 31(5):874-879.

20. Sorensen K, Van den Broucke S, Pelikan JM, Fullam J, Doyle G, Slonska Z, Kondilis B, Stoffels V, Osborne RH, Brand H, HLS-EU Consortium: Measuring 
health literacy in populations: illuminating the design and development process of the European Health Literacy Survey Questionnaire (HLS-EU-Q). BMC Public Health 2013, 13:948.

21. HLS-EU Consortium: Comparative Report of Health Literacy in Eight EU Member States. The European Health Literacy Survey HLS-EU; 2012 [http://www.health-literacy.eu]

22. Paasche-Orlow MK, Parker RM, Gazmararian JA, Nielsen-Bohlman LT, Rudd RR: The prevalence of limited health literacy. J Gen Intern Med 2005, 20(2):175-184.

23. Barber MN, Staples M, Osborne RH, Clerehan R, Elder C, Buchbinder R: Up to a quarter of the Australian population may have suboptimal health literacy depending upon the measurement tool: results from a population-based survey. Health Promot Int 2009, 24(3):252-261.

24. Protheroe J, Nutbeam D, Rowlands G: Health literacy: a necessity for increasing participation in health care. Br J Gen Pract 2009, 59(567):721-723.

25. Ishikawa $H$, Yano E: Patient health literacy and participation in the health-care process. Health Expect 2008, 11(2):113-122.

26. Jordan JE, Osborne RH, Buchbinder R: Critical appraisal of health literacy indices revealed variable underlying constructs, narrow content and psychometric weaknesses. J Clin Epidemio/ 2011, 64(4):366-379.

27. Christensen Al, Ekholm O, Glumer C, Andreasen AH, Hvidberg MF, Kristensen PL, Larsen FB, Ortiz B, Juel K: The Danish National Health Survey 2010. Study design and respondent characteristics. Scand J Public Health 2012, 40(4):391-397. 31.

28. Särndal C, Lundström S: Estimation in surveys with nonresponse. Chichester: John Wiley \& Sons Ltd; 2005.

29. Hawkins M, Osborne R: Questionnaire Translation and Cultural Adaptation Procedure. Deakin Population Health Strategic Research Centre, Deakin University; 2010 [http://www.deakin.edu.au/health/research/phi/Translation\% 20Procedure\%20version\%203.1\%20for\%20HLQ\%20FINAL\%20130422.pdf]

30. Statistics Denmark: Indvandrere i Danmark 2012 (Immigrants in Denmark 2012). Statistics Denmark; 2012 [http://www.dst.dk/pukora/epub/upload/ 16601/indv.pdf]

31. Arendt JN, Jensen BT, Nexøe J, Müntzberg G, Sørensen J: Low income and number of contacts with general practice. Ugeskrift for laeger - LA Danish 2010, 172(21):1591

32. Danish Ministry of Health: Lov om videnskabsetisk behandling af sundhedsvidenskabelige forskningsprojekter (Law on ethical conduct in health science), Lovtidende A, chapter 4, §14, section 2. 2011.

33. Rosdahl A, Fridberg T, Jacobsen V, Jørgensen M: Færdigheder i læsning, regning og problemløsning med IT i Danmark (Literacy, Numeracy and Problem Solving in IT in Denmark).The Danish National Centre for Social Research; 2013 [http:// www.oecd.org/site/piaac/Denmark_1328-Danskernes-kompetencer.pdf]

34. OECD: OECD Skills Outlook 2013: First Results from the Survey of Adult Skills. OECD Publishing; 2013 [http://dx.doi.org/10.1787/9789264204256-en]

35. van der Heide I, Rademakers J, Schipper M, Droomers M, Sorensen K, Uiters E: Health literacy of Dutch adults: a cross sectional survey. BMC Public Health 2013, 13:179-2458. 13-179.

36. van der Heide I, Wang J, Droomers M, Spreeuwenberg P, Rademakers J, Uiters E: The relationship between health, education, and health literacy: results from the Dutch adult literacy and life skills survey. $J$ Health Commun 2013, 18(Suppl 1):172-184.

37. Galobardes B, Shaw M, Lawlor DA, Lynch JW, Davey Smith G: Indicators of socioeconomic position (part 1). J Epidemiol Commun Health 2006, 60(1):7-12.

38. Nutbeam D: The evolving concept of health literacy. Soc Sci Med 2008, 67(12):2072-2078.

39. Andrulis DP, Brach C: Integrating literacy, culture, and language to improve health care quality for diverse populations. Am J Health Behav 2007, 31(Suppl 1):122-133.

40. Edwards M, Wood F, Davies M, Edwards A: 'Distributed health literacy': longitudinal qualitative analysis of the roles of health literacy mediators and social networks of people living with a long-term health condition. Health Expect 2013, 32. in press.

41. Thomson S, Osborn R, Squires D, Jun M: International Profiles of Health Care Systems. The Commonwealth Fund; 2013 [http://www.commonwealthfund. org/ /media/Files/Publications/Fund\%20Report/2012/Nov/1645_Squires_intl_ profiles_hlt_care_systems_2012.pdf]

42. Berkman ND, Sheridan SL, Donahue KE, Halpern DJ, Viera A, Crotty K, Holland A, Brasure M, Lohr KN, Harden E, Tant E, Wallace I, Viswanathan M:
Health literacy interventions and outcomes: an updated systematic review. Evid Rep Technol Assess (Full Rep) 2011, 199:1-941.

43. Chew LD, Griffin JM, Partin MR, Noorbaloochi S, Grill JP, Snyder A, Bradley KA, Nugent $S M$, Baines $A D$, Vanryn M: Validation of screening questions for limited health literacy in a large VA outpatient population. $J$ Gen Intern Med 2008, 23(5):561-566.

doi:10.1186/1471-2458-14-1095

Cite this article as: Bo et al:: National indicators of health literacy: ability to understand health information and to engage actively with healthcare providers - a population-based survey among Danish adults. BMC Public Health 2014 14:1095.

\section{Submit your next manuscript to BioMed Central and take full advantage of:}

- Convenient online submission

- Thorough peer review

- No space constraints or color figure charges

- Immediate publication on acceptance

- Inclusion in PubMed, CAS, Scopus and Google Scholar

- Research which is freely available for redistribution

Submit your manuscript at www.biomedcentral.com/submit
C BioMed Central 